\title{
Patient-Derived Xenografts of Non Small Cell Lung Cancer: Resurgence of an Old Model for Investigation of Modern Concepts of Tailored Therapy and Cancer Stem Cells
}

\author{
Massimo Moro, ${ }^{1}$ Giulia Bertolini, ${ }^{1}$ Monica Tortoreto, ${ }^{2}$ Ugo Pastorino, ${ }^{3}$ \\ Gabriella Sozzi, ${ }^{1}$ and Luca Roz ${ }^{1}$ \\ ${ }^{1}$ Tumor Genomics Unit, Department of Experimental Oncology and Molecular Medicine, \\ IRCCS Foundation National Cancer Institute, Via Venezian 1, 20133 Milan, Italy \\ ${ }^{2}$ Molecular Pharmacology Unit, Department of Experimental Oncology and Molecular Medicine, \\ IRCCS Foundation National Cancer Institute, Via Venezian 1, 20133 Milan, Italy \\ ${ }^{3}$ Thoracic Surgery Unit, Department of Surgery, IRCCS Foundation National Cancer Institute, Via Venezian 1, 20133 Milan, Italy \\ Correspondence should be addressed to Luca Roz, luca.roz@istitutotumori.mi.it
}

Received 28 December 2011; Accepted 10 January 2012

Academic Editor: Andrea Vecchione

Copyright () 2012 Massimo Moro et al. This is an open access article distributed under the Creative Commons Attribution License, which permits unrestricted use, distribution, and reproduction in any medium, provided the original work is properly cited.

Current chemotherapy regimens have unsatisfactory results in most advanced solid tumors. It is therefore imperative to devise novel therapeutic strategies and to optimize selection of patients, identifying early those who could benefit from available treatments. Mouse models are the most valuable tool for preclinical evaluation of novel therapeutic strategies in cancer and, among them, patient-derived xenografts models (PDX) have made a recent comeback in popularity. These models, obtained by direct implants of tissue fragments in immunocompromised mice, have great potential in drug development studies because they faithfully reproduce the patient's original tumor for both immunohistochemical markers and genetic alterations as well as in terms of response to common therapeutics They also maintain the original tumor heterogeneity, allowing studies of specific cellular subpopulations, including their modulation after drug treatment. Moreover PDXs maintain at least some aspects of the human microenvironment for weeks with the complete substitution with murine stroma occurring only after 2-3 passages in mouse and represent therefore a promising model for studies of tumor-microenvironment interaction. This review summarizes our present knowledge on mouse preclinical cancer models, with a particular attention on patient-derived xenografts of non small cell lung cancer and their relevance for preclinical and biological studies.

\section{Introduction}

The continuously growing body of knowledge about molecular events driving oncogenesis has led to the identification of new potential targets for therapy and has consequently paved the way for the design of targeted compounds, offering potential new tools to improve the clinical outcome of cancer patients. The therapeutic efficacy of this large number of new compounds compared to standard treatments (or in association with them) has to be ultimately assessed through clinical trials [1] but drug development process includes many steps and requires investments both in time and resources as well as the recruitment of patients who agree to take part in human clinical trials. Typically, the developmental plan for a cancer chemotherapy agent involves sequential steps such as in vitro studies to identify the basic properties of the compound; rodent studies to assess its potential activity; pharmacology studies to define drug absorption, distribution, metabolism, and elimination; toxicology studies to define a starting dose for humans [2]. The most expensive steps are associated with preclinical toxicology and pharmacology studies and for this reason it is of primary importance to recognize early in the process compounds unworthy of further development. Many different in vitro 
assays have been used to identify lead compounds as exemplified by the 60 human tumor cell line anticancer drug screen (NCI60) developed by the US National Cancer Institute in the late 1980s as an in vitro drug discovery tool which has proved valuable for high-throughput screens and initial assessment of novel compounds. The subsequent efficacy assessment in rodents, however, plays a crucial role in identifying those drugs which deserve further development. In fact, compounds that showed some activities as anticancer drugs on the NCI60 panel must undergo in vivo studies to be presented to the Drug Development Group as candidates for NCI development or licensing [3] highlighting the need for animal models that closely recapitulate human disease for efficient drug development.

\section{Mouse Preclinical Cancer Models}

In general the reliability of rodent-based preclinical cancer models can be questioned because of their imperfect correlation with human clinical outcomes [4]. However, although some drugs that show activity against human tumor xenografts (XGs) have failed to show activity in human clinical trials, many of the clinically approved drugs in use today have demonstrated and continue to demonstrate activity in a variety of preclinical models [4-7]. In addition, it is noteworthy to remember that mouse models are the most valuable tool for preclinical evaluation of novel therapeutic strategies in cancer primarily because humans and mice are genetically closely related and consequently the major signaling pathways are conserved between the two species. The mouse is also small and relatively inexpensive to breed and house. Moreover, the mouse germ line can be modified to generate many different mouse strains modeling different aspects of cancerogenesis. In the early 1980s, immunologically compromised mice, capable of supporting growth of human tumors, became more widely available [8], resulting in the development of human tumor xenografts models which represent, together with genetically engineered models (GEMs), the main categories of preclinical cancer models used for current preclinical efficacy studies. Distinct advantages and disadvantages of the different models are summarized in Table 1.

2.1. Genetically Engineered Mice (GEMs). A genetically modified mouse is a mouse whose genome has been altered by genetic engineering techniques. The genome of these mice is altered in genes known to be involved in malignant transformation, that can be mutated, deleted, or overexpressed; subsequently, the effect of altering these genes is studied over time to evaluate the effect on tumorigenesis (both spontaneous or induced) and eventually therapeutic responses may be followed in vivo [9-11].

There are two basic technical approaches to produce genetically modified mice. The first involves pronuclear injection into a single cell of the mouse embryo, where it will randomly integrate into the mouse genome [12] generating a transgenic mouse. The second approach involves modifying embryonic stem cells with a DNA construct containing
DNA sequences homologous to the target gene [13]. This method is used to manipulate a single gene, in most cases "knocking out" the target gene, although more subtle genetic manipulation can occur (e.g., only changing single nucleotides).

The peculiar features of GEMs (germline alterations) make them particularly suitable to follow tumor development from early time points. Moreover, the development of a tumor resembling the human counterpart in a genetically modified mouse can be very useful for refined studies of relationship with the microenvironment, that can also be manipulated in mice trough specific genetic manipulations or using bone marrow transplantation. Furthermore, the possibility to cross GEMs with other inbred mice strains provides the opportunity to investigate the role of different genetic alterations in tumor development. It should however be stressed that GEMs tumors are murine tumors and although they might present characteristics closely resembling the human counterpart, they could never fully recapitulate a human tumor $[14,15]$. Moreover, the number of modified genes is usually too limited to be representative of the heterogeneity of human solid tumors and the development of a genetically modified mouse strain is very costly and time consuming.

2.2. Mouse Xenograft Models. Xenotransplantation is defined as any procedure that involves the transplantation of living cells, tissues or organs from one species to another.

The growth of human tumors in a different species (e.g., mouse) requires immunodeficiency in the host animal to prevent rejection of the transplanted foreign tissues. There are many strains of genetically determined immunodeficient mice containing single mutations (e.g., nude, scid, beige, xid, rag-1 null, rag-2 null) or combined mutations, (e.g., bg/nu, $\mathrm{bg} / \mathrm{nu} / \mathrm{xid}$, nude/scid, nod/scid) available for cancer research [16-18]. These strains have different immunological impairment and the availability of more permissive mouse strains can strongly increase the efficiency of xenotransplantation; however severely immunocompromised mice also have a higher cost and their manipulation can be extremely demanding requiring controlled husbandry environment and highly trained personnel. It is therefore mandatory to choose the immunocompromised strain with the best "efficiency of transplantation/costs" ratio for each application.

Successful xenografting of human tumors into nude mice was first reported in the late 1960s $[19,20]$. Nude mouse models are now extensively used in the development of potential anticancer drugs and studies of tumor biology and mice with severe combined immunodeficiencies (e.g., SCID, beige, xid) have widened the spectrum of possible models and enabled engraftments of human tumors that were previously difficult to implant.

Tumor xenograft (XG) models can be broadly derived either from the injection in immunocompromised mice of human established tumor cell lines or from direct implant of patient's tumor fragments. This latter model has been referred to by different researchers as "tumorgraft," "primary-tumor xenograft," or "patient-derived xenograft" to 
TABLE 1: Main advantages and disadvantages of GEM, XG, and PDX mouse models.

\begin{tabular}{|c|c|c|}
\hline & Advantages & Disadvantages \\
\hline $\begin{array}{l}\text { Genetically engineered mice } \\
\text { (GEM) }\end{array}$ & $\begin{array}{l}\text { (i) Studies on defined mutations } \\
\text { (ii) Possibility to follow tumor development from } \\
\text { early time points } \\
\text { (iii) Tumor microenvironment is representative of } \\
\text { the studied tumor } \\
\text { (iv) Potential analysis of effects of mutations in } \\
\text { many genetic backgrounds by using a variety of } \\
\text { mouse strains }\end{array}$ & $\begin{array}{l}\text { (i) Limited number of genes (usually not } \\
\text { representative of the heterogeneity of the tumor) } \\
\text { (ii) Development costly and time consuming } \\
\text { (iii) Tumor development in animals slow and } \\
\text { variable } \\
\text { (iv) Both, tumor and microenvironment are } \\
\text { murine }\end{array}$ \\
\hline $\begin{array}{l}\text { Xenograft } \\
(\mathrm{XG})\end{array}$ & $\begin{array}{l}\text { (i) Allows a rapid analysis of response to a } \\
\text { therapeutic regimen } \\
\text { (ii) Source of material virtually unlimited for } \\
\text { immortal cell lines } \\
\text { (iii) Can predict drug response of human patient's } \\
\text { tumor }\end{array}$ & $\begin{array}{l}\text { (i) Human tumor microenvironment is not } \\
\text { represented } \\
\text { (ii) Orthotopic implant is often technically } \\
\text { complicated } \\
\text { (iii) Cells can undergo genetic modification as } \\
\text { well as subpopulation rearrangements when } \\
\text { cultured }\end{array}$ \\
\hline $\begin{array}{l}\text { Patient-derived xenograft } \\
\text { (PDX) }\end{array}$ & $\begin{array}{l}\text { (i) Provides a realistic representation of the } \\
\text { heterogeneity of tumor cell subpopulations } \\
\text { (ii) Can predict drug response of human patient's } \\
\text { tumor } \\
\text { (iii) Stromal component is representative of the } \\
\text { parental tumor in the initial passages }\end{array}$ & $\begin{array}{l}\text { (i) Orthotopic implant is often technically } \\
\text { complicated } \\
\text { (ii) Surgical fragments must be processed rapidly } \\
\text { (iii) Limited source of original material }\end{array}$ \\
\hline
\end{tabular}

point out the difference from conventional "xenografts" generally intended as injection of suspensions of tumor cell lines. For clarity throughout this paper we will refer to the implantation of patient's tumor fragments as "patientderived xenograft" (PDX), while the whole cohort of mice carrying the expansion of tumor fragments derived from the same patient will be defined as "xenopatients" (XP).

In general, established tumor cell lines have a much higher take rate when inoculated as a suspension into nude mice than human solid tumors of the same histological type that are transplanted directly from the patient [21]. On the other hand, culturing tumor cells could lead to changes in gene expression [22] as well as rearrangements in the composition of the cellular populations that constitute the original human tumor resulting in the xenotransplant of a cellular population that is not fully representative of the original tumor heterogeneity. Moreover, studies on XGs of human cell lines to test drug responses do not often correlate with clinical activity in patients [23], especially when cells are injected subcutaneously. By contrast, when cells are used as an orthotopic XG, there is a stronger predictive response value, especially when a clinically relevant drug dosage is used [23-25].

Implantation of either cells or tumor fragments is usually performed subcutaneously rather than orthotopically, because of the technical difficulties often encountered in these assays. This can raise doubts about the development of tumor vasculature and tumor-stroma interactions compared to those observed in the human tumor of origin. Interestingly, as will be discussed more in detail later in this paper, PDXs maintain the whole structure of the human parental tumor during the initial passages in mouse making them suitable also for microenvironment studies.

\section{Patient-Derived Xenografts}

Direct implantation of small tumor fragments in immunocompromised mice can result in growth of patient-derived xenografts that accurately reproduce the heterogeneity of human cancers, especially if a large set of a different tumor subtypes is available. These models can be serially propagated in mice by subsequent passaging as tissue explants and the use of standardized procedures for the assessment of therapeutic efficacy of different drugs using PDXs allows a rapid evaluation of combined therapies on a relatively large set of tumors. However, in order to be relevant as a useful tool for preclinical studies, a PDX panel should accurately reproduce human cancers representing their various subcategories and a particular PDX should accurately reproduce the original patient's tumor. Furthermore, there should be a high correlation between preclinical and clinical results in terms of therapeutic efficacy, with the gold standard being represented by PDXs that closely mimic (or even predict) the clinical response of the patient's tumor they derive from. All these features have been recently investigated in PDXs from different human tumors resulting in the observation that PDXs might represent a suitable model for preclinical studies intended as a "quick" evaluation of the response to different therapeutic treatments. Although the rapidity of this kind of approach is relative, being dependent on the rate of implant of the tumor, it can nonetheless give very relevant information in different clinical settings. On the one hand, while the models are being established and the patients are undergoing first-line treatment, it is possible to acquire proof-of-concept information on relative sensitivity or resistance to the different regimens to be correlated with the molecular features of the PDXs, while on the other it is 
possible to imagine an immediate clinically relevant use of these models in selecting the best second line treatment for a specific patient.

Hidalgo et al. recently reported their results obtained with various advanced solid tumors resected from 14 patients, propagated in immunodeficient mice, and treated with 63 drugs in 232 treatment regimens. They showed an overall remarkable correlation between drug activity in the model and clinical outcome, both in terms of resistance and sensitivity. Their results emphasize the relevance of PDX panels in preclinical studies of anticancer treatments. The treatments they selected for each patient, based on the results obtained with the correspondent PDX, would not have been the first choice for a second- or third-line treatment, nonetheless the objective response rate they obtained was $88 \%$ for treatments deemed effective by the model and tested in the patient, with 11 of 14 patients achieving a partial response. This work highlights that personalized PDXs can be used to investigate drug response with the final aim to select best personalized treatments to increase the response rate of the patients [26].

Another preclinical setting in which PDXs can be extremely relevant is represented by the evaluation of the potential of new drugs in cancer treatment. Taking advantage from a panel of PDX which faithfully represents the heterogeneity of a cancer type, new drugs can be tested and can lead to the identification of the best treatment regimen for a specific subtype of tumor as well as to the identification of new biological pathways involved in the development of the tumor.

Encouraging results, which emphasize the potential of this kind of preclinical model, were recently obtained with metastatic colorectal cancer (mCRC) by Bertotti et al. that established a large PDX cohort from 85 patient-derived mCRC samples. Firstly, they validated the robustness of the cohort of xenopatients showing that they responded to the anti-EGFR antibody Cetuximab with analogous rates compared to those observed in the clinic. Then, they stratified their PDXs in responders and nonresponders and found an enrichment of tumors with HER2 amplification in Cetuximab-resistant KRAS/NRAS/BRAF/PIK3CA wild-type cases (36\% versus $2.7 \%$ in unselected tumors). This approach allowed them to discover that inhibition of HER2 in combination with anti-EGFR antibodies induced overt, longlasting tumor regression in Cetuximab-resistant colorectal cancers, an observation worthy of further clinical investigation [27].

Cetuximab response was also investigated in 79 different PDXs generated from colon, gastric, head and neck, lung and mammary cancer, leading to the identification of MET activation as a mechanism for drug resistance. In particular these models were used for an in-depth analysis of different molecular characteristics of the tumors, including EGFR expression and activation, mutational status of KRAS, BRAF, and NRAS, expression of EGFR ligands and activation of HER3 (ErbB3), and hepatocyte growth factor receptor (MET). High expression and activation of EGFR and its ligands epiregulin or amphiregulin were identified as positive predictive factors regulating Cetuximab response, whereas negative factors were markers for downstream pathway activation independent of EGFR. Interestingly, overexpression due to gene amplification and strong activation of MET was specifically identified in Cetuximab resistant NSCL adenocarcinomas [28].

\section{PDX Models of Non Small Cell Lung Cancer}

4.1. Establishment and Preclinical Relevance. Due to its high incidence and mortality lung cancer is the leading cause of cancer deaths worldwide. The two major forms of lung, cancer are non small cell lung cancer (NSCLC, about 85\% of all lung cancer) and small cell lung cancer (SCLC, about $15 \%)$. NSCLC can be divided into three major histological subtypes: squamous cell carcinoma, adenocarcinoma, and large cell carcinoma. Smoking is the main etiological factor for all types of lung cancer although it is most strongly linked with SCLC and squamous cell carcinoma; on the other hand adenocarcinoma is the most common type of lung tumor in patients who have never smoked $[29,30]$. Currently most NSCLC are diagnosed in advanced stages where five-year survival rates are less than $10 \%$. Since available therapies only provide a modest survival benefit a better understanding of the molecular basis of the disease and novel therapies are needed to significantly improve patients' outcome. The establishment of PDXs from lung cancer specimens has therefore been investigated by us and others both as a source of therapeutically relevant information and as a supply of precious biological material.

After approval from the internal review board, samples of primary non small cell lung cancer are obtained from patients undergoing surgical resection. Each sample is immediately cut in small pieces $\left(25-30 \mathrm{~mm}^{3}\right)$ in antibioticcontaining buffer (PBS 1x, $200 \mathrm{U} / \mathrm{mL}$ penicillin, $200 \mathrm{ug} / \mathrm{mL}$ streptomycin) and implanted subcutaneously in the flank region of 4 to 6 weeks old female nude or SCID (severe combined immunodeficient) anesthetized mice. In detail, animals are anesthetized with an intraperitoneal injection of ketamine/xylazine/saline mixture $(20: 2.5: 77.5 \mathrm{v} / \mathrm{v} / \mathrm{v})$ at a dose of $10 \mathrm{~mL} / \mathrm{kg}$ body weight. Fragments are then implanted using a trocar gauge and mice are maintained in rooms with constant temperature and humidity (Figure 1). Tumor size is evaluated once per week by caliper measurements and the relative tumor weight is estimated, assuming the PDX as an ellipsoid with a specific density of $1.0 \mathrm{~g} / \mathrm{cm}^{3}$, through the $\left(\mathrm{Lxl}^{2}\right) / 2(\mathrm{mg})$ formula, where $\mathrm{L}$ is the longest diameter and 1 the shortest [31].

Typically, after a variable lag time, the tumor begins to grow exponentially until it reaches a plateau level where the growth slows down; it is important to transfer the tumor to a new mouse in the period of exponential growth, mostly not only for ethical reasons (tumor charge must never exceed $1 / 10$ of the mouse body weight), but also because the probability of necrotic areas within the PDX increases when it reaches a higher mass. Highly necrotic tumors are difficult to propagate and great care should be taken to avoid this occurrence. Successive rounds of expansion from donor to 


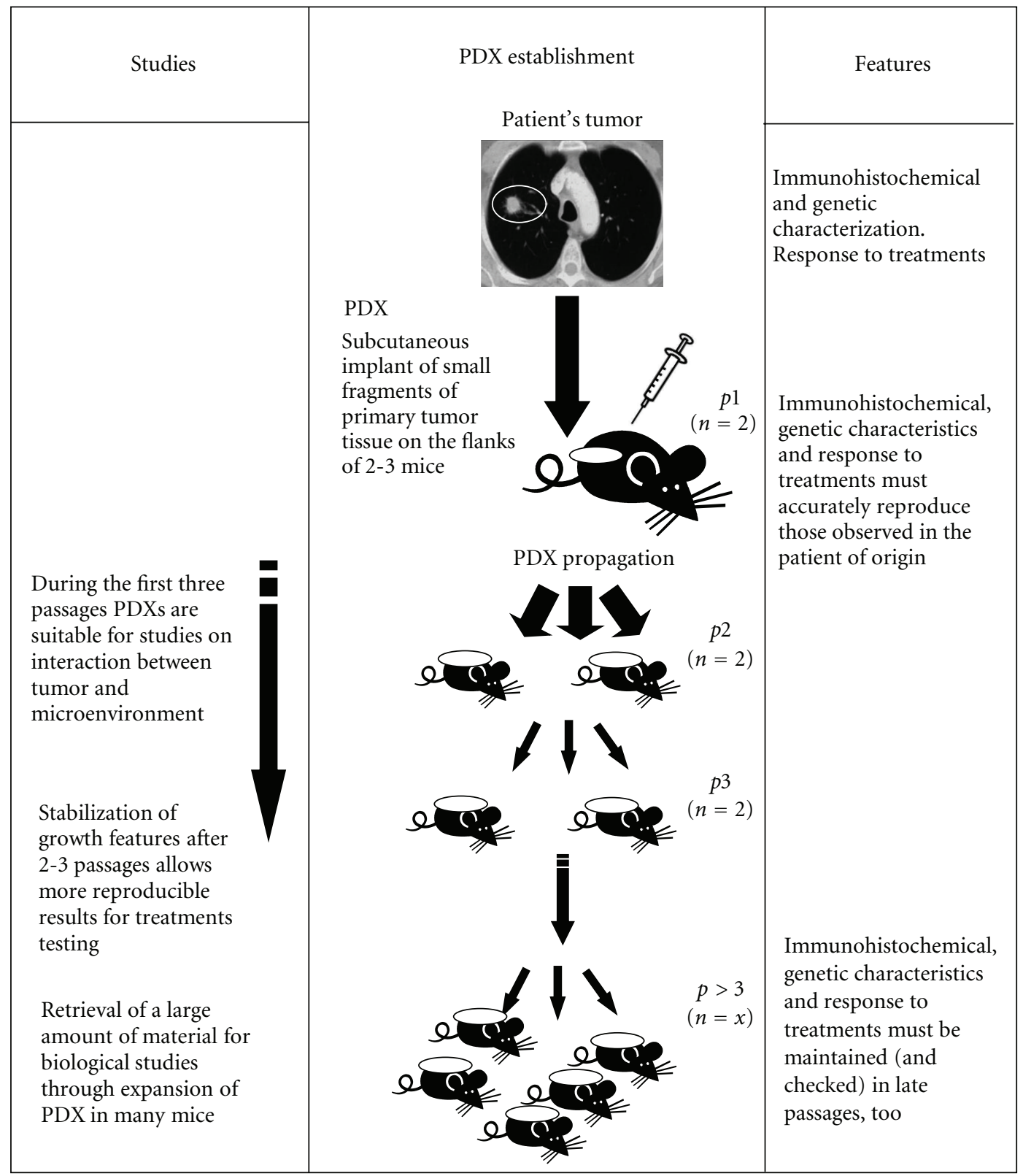

FIGURE 1: Flow-chart of establishment of patient-derived xenografts. Fragments of primary tumor samples are initially implanted subcutaneously in both flanks of 1-2 immunocompromised mice, depending on tissue availability $(p 0)$. When tumors reach the exponential growth phase they are removed from donor mice, reduced into fragments, and serially transplanted in new recipient mice ( $p 1)$. For investigational purposes (i.e., drug treatments or subpopulation analysis) PDXs can be expanded in a higher number of mice in order to obtain statistical relevant results or sufficient biological material for analysis, respectively. ( $p$ : sequential passage in immunocompromised mouse; $n$ : number of mice.)

recipient mice can produce a sizable cohort of xenopatients suitable for pharmacological experiments.

Each model has its specific characteristics when implanted subcutaneously in different immunodeficient mouse strains, so it is very important to monitor tumor progression using parameters that can be useful in subsequent experiments designed to evaluate the effects of different compounds on individual PDXs.

The first parameter that should be evaluated is the percentage of the tumor take in mice. Usually, for lung cancers about $30-40 \%$ of the patient-derived tumors are successfully implanted subcutaneously in nude mice. Interestingly, it has been reported a correlation between the ability to form PDXs and the risk of disease recurrence in earlystage NSCLC [32]. In this study $40 \%$ of NSCLC implanted resulted in a successful PDX with KRAS-mutated tumors engrafting more efficiently than EGFR-mutated ones and positive engraftment correlating with shorter disease-free survival in a multivariate analysis including age, sex, stage, and mutations. 
Other useful parameters are related to the growth characteristics of the PDX. As previously described one feature defining PDX is its lag time before exponential growth that can be stable or variable during subsequent passages of the tumor. In our experience the lag time is generally higher for the first two-three passages and then reaches a steady state level, probably due to the progressive substitution of the original human tumor stroma with murine stroma.

Beyond the lag time, the parameters to follow during PDX establishment are specifically related to the growth characteristics of the model: growth rate, doubling time, time before implantation in another mouse are all useful tools to catalogue a panel of PDX in groups with similar characteristics. Mouse general health should also be monitored during the whole process of PDX implantation. Indeed we have observed that some tumors can induce a general health's worsening when implanted subcutaneously in mice in a way reminiscent to the cachexia-inducing potential of some human tumors. In these situations, if the growth of the model can be supported in a different strain, transferring the PDX in a less immunocompromised mouse strain (i.e., from SCID to nude mice) can clear up these kind of cachexiarelated issues resulting in a fast-growing "healthy" PDX. This could be suggestive of a higher aggressiveness of tumors with such features, that are probably too aggressive to be sustained by an organism with a deeply compromised immune system, but further data are needed to confirm this observation.

According to our experience, PDXs can maintain the same characteristics of the human primary tumor for several passages (up to 20), highlighting their value as a resource for multiple studies [33]. Since it can be very expensive and unpractical to continuously passage PDXs in mice it is therefore useful to set up a stock of frozen PDX samples. A good practice is to store samples from earlier passage and then other samples every ten-fifteen passages. To obtain successful thawing of preserved samples, freshly processed tissue fragments should be immediately frozen in a solution of $90 \%$ fetal bovine serum and 10\% DMSO and preserved at $-80^{\circ} \mathrm{C}$.

Also for lung cancer the relevance of PDXs for the evaluation of new therapeutic strategies has been reported. Patientderived NSCLC PDXs have been established by Fichtner and collaborators in order to identify predictive biomarkers. Starting from 102 surgically resected early stage (T2/T3) NSCLC specimens, they set up 25 transplantable PDXs. They showed how, in early passages, PDXs maintain a high degree of similarity with the original clinical tumor sample with regard to histology, immunohistochemistry and mutational status. Also the chemotherapeutic responsiveness of the PDXs panel resembled the clinical situation. Interestingly, they observed a correlation between KRAS mutations and Erlotinib resistance but no correlation between anti-EGFR therapy and mutations in EGFR or p53. Moreover, after treatment with Cetuximab, a down regulation of EGFR was observed in 2 of 6 sensitive PDXs but in none of the resistant PDXs [34].

The same group used 22 well-characterized NSCLC PDX to support the clinical development of the anticancer drug Sagopilone (a fully synthetic low molecular weight analogue of epothilone) involved in an integrative preclinical phase II design. According to clinical trial criteria, 64\% (14 of 22) of lung cancer PDXs were sensible to Sagopilone. Interestingly, tumors with wild-type TP53 as well as with a high expression of genes involved in cell adhesion/angiogenesis were more likely to be resistant to Sagopilone. Therefore a combination of Sagopilone with Bevacizumab and Sorafenib, drugs targeting vascular endothelial growth factor signaling, was tested in Sagopilone-resistant models, restoring antitumor activity [35].

These data confirm the potential relevance of wellannotated PDX panels to stratify the observed responses on the basis of specific molecular alterations. This holds great promise not only for evaluating conventional therapeutics, but also for testing novel drug candidates as well as to identify rationale combination therapies to be tested in clinical trials.

At the other end, for studies on personalized chemotherapy, intended as a "quick" evaluation of the putative treatment response of the individual patient, lung cancer and in particular NSCLC-derived PDXs appear currently not very suitable due to their relative low take rate (30-40\%) if implanted subcutaneously and also for the slow growth rate (often several months). An interesting approach to bypass these issues has been proposed by Dong and colleagues who established PDX from 32 untreated, completely resected patients' NSCLCs. They obtained an engraftment rate of $90 \%$ implanting small pieces of tumors under the renal capsules of NOD/SCID mice. Treating these xenopatients with either cisplatin + vinorelbine, cisplatin + docetaxel, or cisplatin + gemcitabine they quickly assessed (in 6-8 weeks) the chemosensitivity of patients' cancers and selected the most effective regimen. Only 16 of the 32 tumors provided sufficient tissue for testing all three chemotherapeutic regimens, but it is noteworthy that 11 patients had adjuvant therapy that matched the regimen used for the corresponding PDX with good concordance between the results obtained in the animal model and clinical response, in particular in relation to resistance to conventional therapy (six of seven patients who developed recurrence/metastasis during followup were nonresponsive in mice) highlighting the need for novel strategies [36]. However, the technique of engraftment under the renal capsules is certainly more difficult compared to subcutaneous implantation of the tumor fragments and NOD/SCID mice are more costly and also more difficult to breed than SCID or nude mice, which are usually utilized to set up a PDX model.

Setting up a panel of PDX that can be used to test new agents is costly and time and resource consuming, moreover the selection of compounds for advancement to human clinical trials is based, in part, on in vivo efficacy studies. It is therefore of primary importance to validate the efficacy of the PDX panel in their representation of the human tumor. As previously mentioned, PDXs must show histological and genetic characteristics similar to patient's tumors they are derived from. These characteristics should be checked to remain unvaried after several passages in mouse, too. Furthermore, the panel of PDXs available for a study must be representative of the heterogeneity of the selected cancer type as well as possible. In the case of lung 
cancers it is mandatory to obtain a PDX panel covering all the three major histological subtypes previously described (i.e., squamous cell carcinoma, adenocarcinoma, and large cell lung cancer; whereas a study on NSCLC in nonsmokers should be mainly derived from adenocarcinomas) and to evaluate the response to the common chemotherapeutic(s) utilized in clinic (e.g., cisplatin), that should mimic the patients' response tumor. These data, together with histological and genetic characteristics, could provide the basis for a classification of the PDXs in groups of tumors with similar features and similar behavior and will be very useful in testing the activity of new compounds for NSCLC.

4.2. Relevance for Biological Research. The availability of fresh tissue from primary human lung cancers expanded in vivo as PDXs also constitutes an important repository of biological material for different studies ranging from investigation of tumor-microenvironment interaction to evaluation of tumor heterogeneity and investigation of dynamics of different subpopulations during therapy.

Investigations have been carried out to define the preservation of tissue architecture in PDXs, including stroma components and resulted in the observation of a remarkable stability of the models even when murine cells have substituted the human counterpart $[37,38]$. On the other hand the presence of human stroma in early passages of PDXs can be initially exploited to study interaction between tumor cells and microenvironment. In particular studies on tumor microenvironment on PDXs of NSCLC at early passages have been carried out by Simpson-Abelson and collaborators. They implanted nondisrupted pieces of primary human lung tumor in severely immunodeficient mouse (NOD/SCID IL2Rgamma null mice) obtaining PDXs in which tissue architecture, including tumor-associated leukocytes, stromal fibroblasts, and tumor cells were preserved for prolonged periods. They observed that plasma cells remained functional in PDX-bearing mice, as evidenced by production of human immunoglobulins, for up to 9 weeks after engraftment. Moreover, tumor-associated T cells were found to migrate from the microenvironment of the PDX to the lung, liver, and primarily to the spleen at 8 weeks post engraftment. These data confirmed the relevance of the PDX model in studying tumor and tumor-stromal cell interactions in situ [39]. Interestingly the same architecture was not maintained when tissue fragments were implanted in CB17-scid mice, indicating once more the need to select the appropriate model for different biological investigations.

PDXs can also be very useful as a source of material for studies on tumor subpopulations and their modulation after drug treatments, leading to a more comprehensive knowledge of tumor development as well as mechanisms of drug resistance. Foci of resistant cells have been identified after cisplatin + vinorelbine, cisplatin + docetaxel, or cisplatin + gemcitabine treatment in responsive NSCLC PDXs by Dong and collaborators [36]. The authors suggested that these drug-resistant cells could be responsible for tumor recurrence as it frequently occurs in patients after partial or even complete response. This observation fits very well with the hypothesis of the presence of small subpopulations of tumor initiating cells within the tumor. This theory, known as the cancer stem cell (CSC) theory has been proposed to explain tumor heterogeneity and the carcinogenesis process [40, 41]. Accordingly to this model, tumor can be represented as the result of abnormal organogenesis driven by CSCs, defined as self-renewing tumor cells able to initiate the tumor formation and to maintain tumor heterogeneity $[42,43]$. Cells with features of CSCs have been identified in acute myeloid leukemia [44], glioblastoma [45-47], melanoma $[48,49]$, and different epithelial cancers [50-56].

Two main techniques are generally used to identify tumor initiating cells: (i) prospective flow cytometry-based cell sorting using tissue-specific surface markers or (ii) sphere forming assays in selective cells culture medium. Due to the relative paucity of the cancer stem cell fraction in the tumor population it is highly desirable to have a substantial amount of tumor samples available for thorough investigation but this is not easily obtained from surgical specimens. Since human cancer PDX models are similar to the patients' tumors not only in terms of genetic, immunohistochemical, and microenvironment features but also in terms of heterogeneity of the tumor cellular composition they are a useful model also in studies of subpopulations with cancer stem cell characteristics (Figure 2). As a matter of fact the possibility to expand the patient-derived tumor in immunocompromised mice yields a large amount of material for studies such as flow cytometry-based cell sorting. Using this approach our group has recently been successful in identifying a highly tumorigenic $\mathrm{CD}_{133^{+}}$subpopulation in non small cell lung cancers which displays stem-like features and is spared by cisplatin treatment $[33,57]$.

PDXs as a tool for CSC studies have been also developed for pancreatic cancer. Jimeno et al. showed that the chemotherapeutic treatment of pancreatic PDXs resulted in an increase of CSC markers (ALDH and CD24), in the residual tumor population, supporting the idea of an enhanced chemoresistance of the cancer stem cells subpopulation. Moreover, the authors showed that CSCs targeting could increase the efficacy of conventional treatment. Combining gemcitabine with an inhibitor of the hedgehog pathway, that is fundamental for the maintenance of CSCs, they induced tumor regression and a decrease in CSC fraction [58]. Similar results were obtained with PDXs derived from other cancer types $[59,60]$, suggesting that combination therapy using conventional chemotherapy and drugs against CSC specific targets can lead to better therapeutic results, both in terms of tumor growth as well as in terms or tumor relapse.

Furthermore, subpopulations isolated from expanded PDXs can also be studied in vivo if reinjected in immunocompromised mice and their characteristics in terms of tumorigenicity as well as in term of response to chemotherapeutics can be directly compared with the characteristics of the parental tumor. This gives the possibility to identify treatment resistant subpopulations and to study the tumorigenic potential of prospectively identified putative CSCs. The relevance of these models in identifying and isolating CSC subpopulation has been proved also for other cancer 


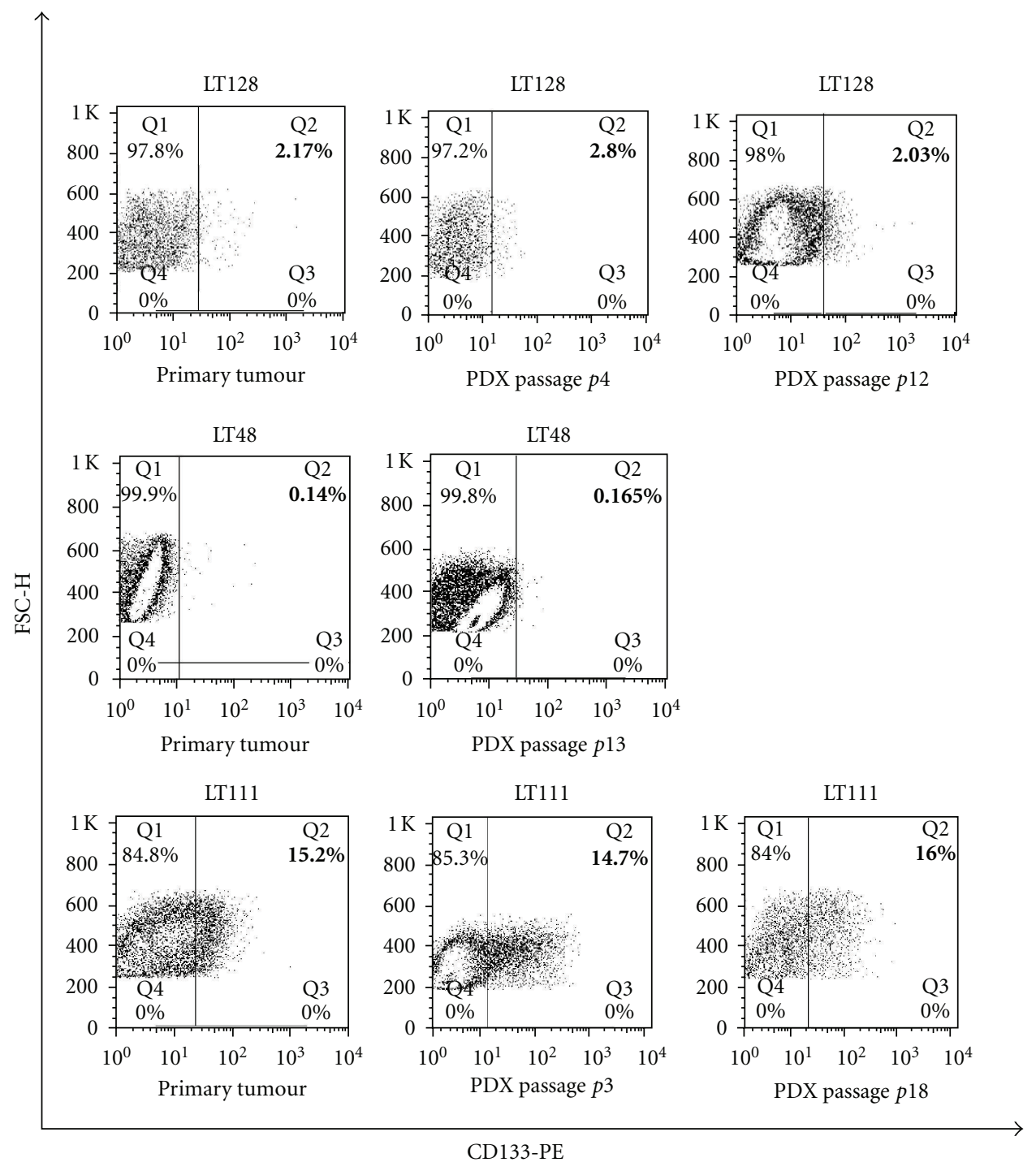

FIGURE 2: Frequency of $\mathrm{CD} 133^{+}$cells in primary tumors is maintained in PDXs during passaging in mice independently of their initial content. Dot plots showing that the percentage of $\mathrm{CD} 133^{+}$cells, previously demonstrated to display stem-like features and to be spared by cisplatin treatment [REF], is similar in the primary tumor and in PDXs. CD $133^{+}$cells levels remain stable also after several passages in immunocompromised mice in PDX models established from low, intermediate and high CD133-expressing tumors (LT48, LT128, LT111 resp.). $\mathrm{p}=$ number of serial transplant in mouse. FACs analysis of CD133 expression was performed with CD133/1-phycoerythrin antibody $(50 \mu \mathrm{g} / \mathrm{mL} ; \mathrm{AC} 133$ clone; Miltenyi Biotech).

types, such as breast cancer [60], colon carcinoma [61], and pancreatic adenocarcinoma $[62,63]$.

\section{Conclusive Remarks}

A growing number of research groups have established panels of patient-derived xenografts, demonstrating their relevance in drug development studies as well as in the discovery of novel predictive biomarkers to be used in the clinical setting.

It is therefore of primary importance to set up panels of PDXs highly representative of the cancer type under investigation and to validate the models through extensive immunohistochemical and genetic characterization to confirm similarities with the human tumor and also in terms of response to common chemotherapeutics to confirm the robustness of the model in testing the efficacy of new treatments. In our experience, growth features of PDX such as lag time and growth rate reach stability after two-three passages in mouse; it is therefore advisable to carry out drug efficacy tests with PDXs that have reached a certain stability in their growth characteristics.

This kind of preclinical model is also exploitable in research fields such as tumor-microenvironment interaction, cancer stem cells identification and modulation after drug treatment. Human cancer PDXs are particularly suitable for this kind of research because they provide a large amount 
of material reproducing the original human tumor and its microenvironment and could therefore be more extensively utilized not only in preclinical studies but also in basic research. However, it is always noteworthy to remember that when using animal models for biological purposes, the guidelines for the welfare and use of animals in cancer research should be always kept in mind and strictly followed [64].

\section{Acknowledgments}

L. Roz and G. Sozzi are supported by grants from Associazione Italiana per la Ricerca sul Cancro (AIRC) and from the European Community Seventh Framework Programme Collaborative Project CURELUNG (to L. Roz).

\section{References}

[1] D. Decaudin, "Primary human tumor xenografted models ("tumorgrafts") for good management of patients with cancer," Anti-Cancer Drugs, vol. 22, no. 9, pp. 827-841, 2011.

[2] S. Venkatesh and R. A. Lipper, "Role of the development scientist in compound lead selection and optimization," Journal of Pharmaceutical Sciences, vol. 89, no. 2, pp. 145-154, 2000.

[3] R. H. Shoemaker, "The NCI60 human tumour cell line anticancer drug screen," Nature Reviews Cancer, vol. 6, no. 10, pp. 813-823, 2006.

[4] S. A. Burchill, "What do, can and should we learn from models to evaluate potential anticancer agents?" Future Oncology, vol. 2, no. 2, pp. 201-211, 2006.

[5] C. A. Carter, C. Chen, C. Brink et al., "Sorafenib is efficacious and tolerated in combination with cytotoxic or cytostatic agents in preclinical models of human non-small cell lung carcinoma," Cancer Chemotherapy and Pharmacology, vol. 59, no. 2, pp. 183-195, 2007.

[6] T. Voskoglou-Nomikos, J. L. Pater, and L. Seymour, "Clinical predictive value of the in vitro cell line, human xenograft, and mouse allograft preclinical cancer models," Clinical Cancer Research, vol. 9, no. 11, pp. 4227-4239, 2003.

[7] L. Q. M. Chow and S. G. Eckhardt, "Sunitinib: from rational design to clinical efficacy," Journal of Clinical Oncology, vol. 25, no. 7, pp. 884-896, 2007.

[8] J. Fogh and B. C. Giovanella, The Nude Mouse in Experimental and Clinical Research, Academic Press, 1978.

[9] H. Castrop, "Genetically modified mice-successes and failures of a widely used technology," Pflugers Archiv European Journal of Physiology, vol. 459, no. 4, pp. 557-567, 2010.

[10] D. J. Wells, "Genetically modified animals and pharmacological research," Handbook of Experimental Pharmacology, vol. 199, pp. 213-226, 2010.

[11] M. A. G. Sosa, R. De Gasperi, and G. A. Elder, "Animal transgenesis: an overview," Brain Structure and Function, vol. 214, no. 2-3, pp. 91-109, 2010.

[12] J. W. Gordon, G. A. Scangos, and D. J. Plotkin, "Genetic transformation of mouse embryos by microinjection of purified DNA," Proceedings of the National Academy of Sciences of the United States of America, vol. 77, no. 12, pp. 7380-7384, 1980.

[13] K. R. Thomas and M. R. Capecchi, "Site-directed mutagenesis by gene targeting in mouse embryo-derived stem cells," Cell, vol. 51, no. 3, pp. 503-512, 1987.
[14] J. H. Lin, "Applications and limitations of genetically modified mouse models in drug discovery and development," Current Drug Metabolism, vol. 9, no. 5, pp. 419-438, 2008.

[15] B. Bolon, "Genetically engineered animals in drug discovery and development: a maturing resource for toxicologic research," Basic and Clinical Pharmacology and Toxicology, vol. 95, no. 4, pp. 154-161, 2004.

[16] R. Clarke, "Animal models of breast cancer: experimental design and their use in nutrition and psychosocial research," Breast Cancer Research and Treatment, vol. 46, no. 2-3, pp. 117-133, 1997.

[17] J. K. Peterson and P. J. Houghton, "Integrating pharmacology and in vivo cancer models in preclinical and clinical drug development," European Journal of Cancer, vol. 40, no. 6, pp. 837-844, 2004.

[18] B. Firestone, "The challenge of selecting the "right" in vivo oncology pharmacology model," Current Opinion in Pharmacology, vol. 10, no. 4, pp. 391-396, 2010.

[19] J. Rygaard and C. O. Povlsen, "Heterotransplantation of a human malignant tumour to "Nude" mice," Acta Pathologica et Microbiologica Scandinavica, vol. 77, no. 4, pp. 758-760, 1969.

[20] B. C. Giovanella, S. O. Yim, J. S. Stehlin, and L. J. Williams, "Development of invasive tumors in the "nude" mouse after injection of cultured human melanoma cells," Journal of the National Cancer Institute, vol. 48, no. 5, pp. 1531-1533, 1972.

[21] B. C. Giovanella, D. M. Vardeman, L. J. Williams et al., "Heterotransplantation of human breast carcinomas in nude mice. Correlation between successful heterotransplants, poor prognosis and amplification of the HER-2/neu oncogene," International Journal of Cancer, vol. 47, no. 1, pp. 66-71, 1991.

[22] V. C. Daniel, L. Marchionni, J. S. Hierman et al., "A primary xenograft model of small-cell lung cancer reveals irreversible changes in gene expression imposed by culture in vitro," Cancer Research, vol. 69, no. 8, pp. 3364-3373, 2009.

[23] R. S. Kerbel, "Human tumor xenografts as predictive preclinical models for anticancer drug activity in humans: better than commonly perceived-but they can be improved," Cancer biology \& therapy, vol. 2, no. 4, pp. S134-S139, 2003.

[24] J. I. Johnson, S. Decker, D. Zaharevitz et al., "Relationships between drug activity in NCI preclinical in vitro and in vivo models and early clinical trials," British Journal of Cancer, vol. 84, no. 10, pp. 1424-1431, 2001.

[25] C. C. Scholz, D. P. Berger, B. R. Winterhalter, H. Henss, and H. H. Fiebig, "Correlation of drug response in patients and in the clonogenic assay with solid human tumour xenografts," European Journal of Cancer, vol. 26, no. 8, pp. 901-905, 1990.

[26] M. Hidalgo, E. Bruckheimer, N. V. Rajeshkumar et al., "A pilot clinical study of treatment guided by personalized tumorgrafts in patients with advanced cancer," Molecular Cancer Therapeutics, vol. 10, no. 8, pp. 1311-1316, 2011.

[27] A. Bertotti, G. Migliardi, F. Galimi et al., "A molecularly annotated platform of patient-derived xenografts ("Xenopatient") identifies HER2 as an effective therapeutic target in cetuximab-resistant colorectal cancer," Cancer Discovery, vol. 1, no. 6, pp. 508-523, 2011.

[28] R. Krumbach, J. Schüler, M. Hofmann, T. Giesemann, H. -H. Fiebig, and T. Beckers, "Primary resistance to cetuximab in a panel of patient-derived tumour xenograft models: activation of MET as one mechanism for drug resistance," European Journal of Cancer, vol. 47, no. 8, pp. 1231-1243, 2011.

[29] S. Sun, J. H. Schiller, and A. F. Gazdar, "Lung cancer in never smokers-a different disease," Nature Reviews Cancer, vol. 7, no. 10 , pp. 778-790, 2007. 
[30] A. Spira, J. Beane, V. Shah et al., "Effects of cigarette smoke on the human airway epithelial cell transcriptome," Proceedings of the National Academy of Sciences of the United States of America, vol. 101, no. 27, pp. 10143-10148, 2004.

[31] J. Plowman, D. J. Dykes, M. Hollingshead, L. Simpson-Herren, and M. C. Alley, "Human tumor xenograft models in NCI drug development," in Anticancer Drug Development Guide: Preclinical Screening, Clinical Trials and Approval, B. Teicher, Ed., pp. 101-125, Humana Press, Totowa, NJ, 1997.

[32] T. John, D. Kohler, M. Pintilie et al., "The ability to form primary tumor xenografts is predictive of increased risk of disease recurrence in early-stage non-small cell lung cancer," Clinical Cancer Research, vol. 17, no. 1, pp. 134-141, 2011.

[33] G. Bertolini, L. Roz, P. Perego et al., "Highly tumorigenic lung cancer $\mathrm{CD} 133^{+}$cells display stem-like features and are spared by cisplatin treatment," Proceedings of the National Academy of Sciences of the United States of America, vol. 106, no. 38, pp. 16281-16286, 2009.

[34] I. Fichtner, J. Rolff, R. Soong et al., "Establishment of patientderived non-small cell lung cancer xenografts as models for the identification of predictive biomarkers," Clinical Cancer Research, vol. 14, no. 20, pp. 6456-6468, 2008.

[35] S. Hammer, A. Sommer, I. Fichtner et al., "Comparative profiling of the novel epothilone, sagopilone, in xenografts derived from primary non-small cell lung cancer," Clinical Cancer Research, vol. 16, no. 5, pp. 1452-1465, 2010.

[36] X. Dong, J. Guan, J. C. English et al., "Patient-derived first generation xenografts of non-small cell lung cancers: promising tools for predicting drug responses for personalized chemotherapy," Clinical Cancer Research, vol. 16, no. 5, pp. 1442-1451, 2010.

[37] E. Marangoni, A. Vincent-Salomon, N. Auger et al., "A new model of patient tumor-derived breast cancer xenografts for preclinical assays," Clinical Cancer Research, vol. 13, no. 13, pp. 3989-3998, 2007.

[38] L. De Plater, A. Laugé, C. Guyader et al., "Establishment and characterisation of a new breast cancer xenograft obtained from a woman carrying a germline BRCA2 mutation," British Journal of Cancer, vol. 103, no. 8, pp. 1192-1200, 2010.

[39] M. R. Simpson-Abelson, G. F. Sonnenberg, H. Takita et al., "Long-term engraftment and expansion of tumor-derived memory T cells following the implantation of non-disrupted pieces of human lung tumor into NOD-scid IL2R $\gamma$ null Mice," Journal of Immunology, vol. 180, no. 10, pp. 7009-7018, 2008.

[40] T. Reya, S. J. Morrison, M. F. Clarke, and I. L. Weissman, "Stem cells, cancer, and cancer stem cells," Nature, vol. 414, no. 6859, pp. 105-111, 2001.

[41] R. Pardal, M. F. Clarke, and S. J. Morrison, "Applying the principles of stem-cell biology to cancer," Nature Reviews Cancer, vol. 3, no. 12, pp. 895-902, 2003.

[42] M. F. Clarke, "Self-renewal and solid-tumor stem cells," Biology of Blood and Marrow Transplantation, vol. 11, no. 2, pp. 14-16, 2005.

[43] M. F. Clarke, J. E. Dick, P. B. Dirks et al., "Cancer stem cellsperspectives on current status and future directions: AACR workshop on cancer stem cells," Cancer Research, vol. 66, no. 19, pp. 9339-9344, 2006.

[44] D. Bonnet and J. E. Dick, "Human acute myeloid leukemia is organized as a hierarchy that originates from a primitive hematopoietic cell," Nature Medicine, vol. 3, no. 7, pp. 730737, 1997.

[45] S. K. Singh, I. D. Clarke, M. Terasaki et al., "Identification of a cancer stem cell in human brain tumors," Cancer Research, vol. 63, no. 18, pp. 5821-5828, 2003.
[46] H. D. Hemmati, I. Nakano, J. A. Lazareff et al., "Cancerous stem cells can arise from pediatric brain tumors," Proceedings of the National Academy of Sciences of the United States of America, vol. 100, no. 25, pp. 15178-15183, 2003.

[47] R. Galli, E. Binda, U. Orfanelli et al., "Isolation and characterization of tumorigenic, stem-like neural precursors from human glioblastoma," Cancer Research, vol. 64, no. 19, pp. 7011-7021, 2004.

[48] D. Fang, T. K. Nguyen, K. Leishear et al., "A tumorigenic subpopulation with stem cell properties in melanomas," Cancer Research, vol. 65, no. 20, pp. 9328-9337, 2005.

[49] T. Schatton, G. F. Murphy, N. Y. Frank et al., "Identification of cells initiating human melanomas," Nature, vol. 451, no. 7176, pp. 345-349, 2008.

[50] M. Al-Hajj, M. S. Wicha, A. Benito-Hernandez, S. J. Morrison, and M. F. Clarke, "Prospective identification of tumorigenic breast cancer cells," Proceedings of the National Academy of Sciences of the United States of America, vol. 100, no. 7, pp. 3983-3988, 2003.

[51] S. A. Bapat, A. M. Mali, C. B. Koppikar, and N. K. Kurrey, "Stem and progenitor-like cells contribute to the aggressive behavior of human epithelial ovarian cancer," Cancer Research, vol. 65, no. 8, pp. 3025-3029, 2005.

[52] A. T. Collins, P. A. Berry, C. Hyde, M. J. Stower, and N. J. Maitland, "Prospective identification of tumorigenic prostate cancer stem cells," Cancer Research, vol. 65, no. 23, pp. 1094610951, 2005.

[53] L. Ricci-Vitiani, D. G. Lombardi, E. Pilozzi et al., "Identification and expansion of human colon-cancer-initiating cells," Nature, vol. 445, no. 7123, pp. 111-115, 2007.

[54] C. A. O'Brien, A. Pollett, S. Gallinger, and J. E. Dick, "A human colon cancer cell capable of initiating tumour growth in immunodeficient mice," Nature, vol. 445, no. 7123, pp. 106$110,2007$.

[55] P. Dalerba, S. J. Dylla, I. K. Park et al., "Phenotypic characterization of human colorectal cancer stem cells," Proceedings of the National Academy of Sciences of the United States of America, vol. 104, no. 24, pp. 10158-10163, 2007.

[56] A. Eramo, F. Lotti, G. Sette et al., "Identification and expansion of the tumorigenic lung cancer stem cell population," Cell Death and Differentiation, vol. 15, no. 3, pp. 504-514, 2008.

[57] G. Bertolini, L. Gatti, and L. Roz, "The "stem" of chemoresistance," Cell Cycle, vol. 9, no. 4, pp. 628-629, 2010.

[58] A. Jimeno, G. Feldmann, A. Suárez-Gauthier et al., "A direct pancreatic cancer xenograft model as a platform for cancer stem cell therapeutic development," Molecular Cancer Therapeutics, vol. 8, no. 2, pp. 310-314, 2009.

[59] T. Hoey, W. C. Yen, F. Axelrod et al., "DLL4 blockade inhibits tumor growth and reduces tumor-initiating cell frequency," Cell Stem Cell, vol. 5, no. 2, pp. 168-177, 2009.

[60] C. Ginestier, S. Liu, M. E. Diebel et al., "CXCR1 blockade selectively targets human breast cancer stem cells in vitro and in xenografts," Journal of Clinical Investigation, vol. 120, no. 2, pp. 485-497, 2010.

[61] S. J. Dylla, L. Beviglia, I. K. Park et al., "Colorectal cancer stem cells are enriched in xenogeneic tumors following chemotherapy," PLoS One, vol. 3, no. 6, Article ID e2428, 2008.

[62] Z. A. Rasheed, J. Yang, Q. Wang et al., "Prognostic significance of tumorigenic cells with mesenchymal features in pancreatic adenocarcinoma," Journal of the National Cancer Institute, vol. 102, no. 5, pp. 340-351, 2010.

[63] M. P. Kim, J. B. Fleming, H. Wang et al., "ALDH activity selectively defines an enhanced tumor-initiating cell population relative to $\mathrm{CD} 133$ expression in human pancreatic 
adenocarcinoma," PLoS One, vol. 6, no. 6, Article ID e20636, 2011.

[64] P. Workman, E. O. Aboagye, F. Balkwill et al., "Guidelines for the welfare and use of animals in cancer research," British Journal of Cancer, vol. 102, no. 11, pp. 1555-1577, 2010. 


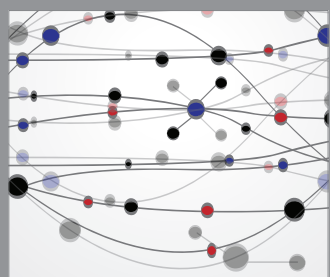

The Scientific World Journal
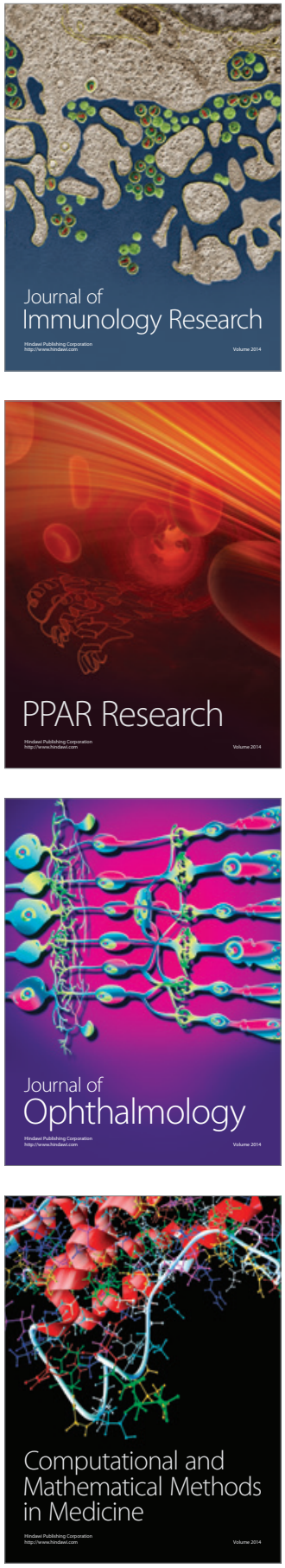

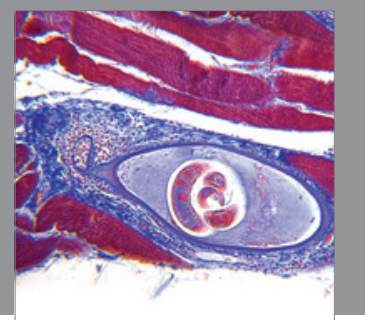

Gastroenterology

Research and Practice
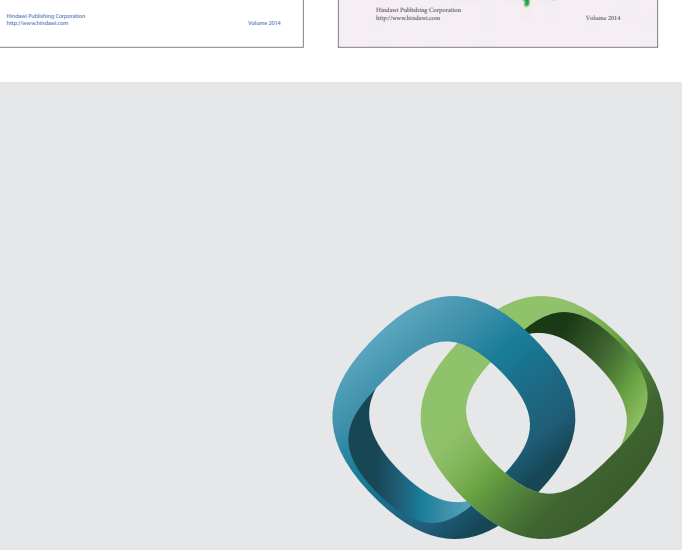

\section{Hindawi}

Submit your manuscripts at

http://www.hindawi.com
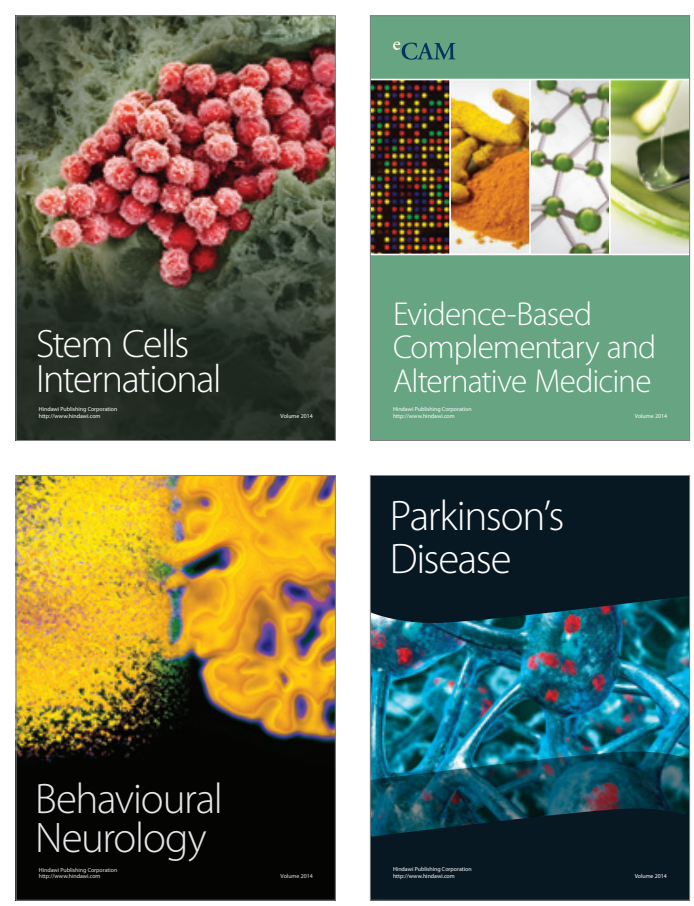

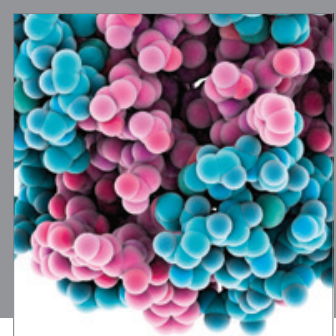

Journal of
Diabetes Research

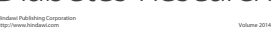

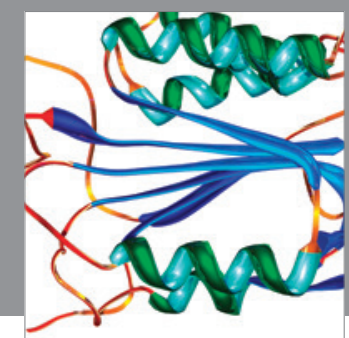

Disease Markers
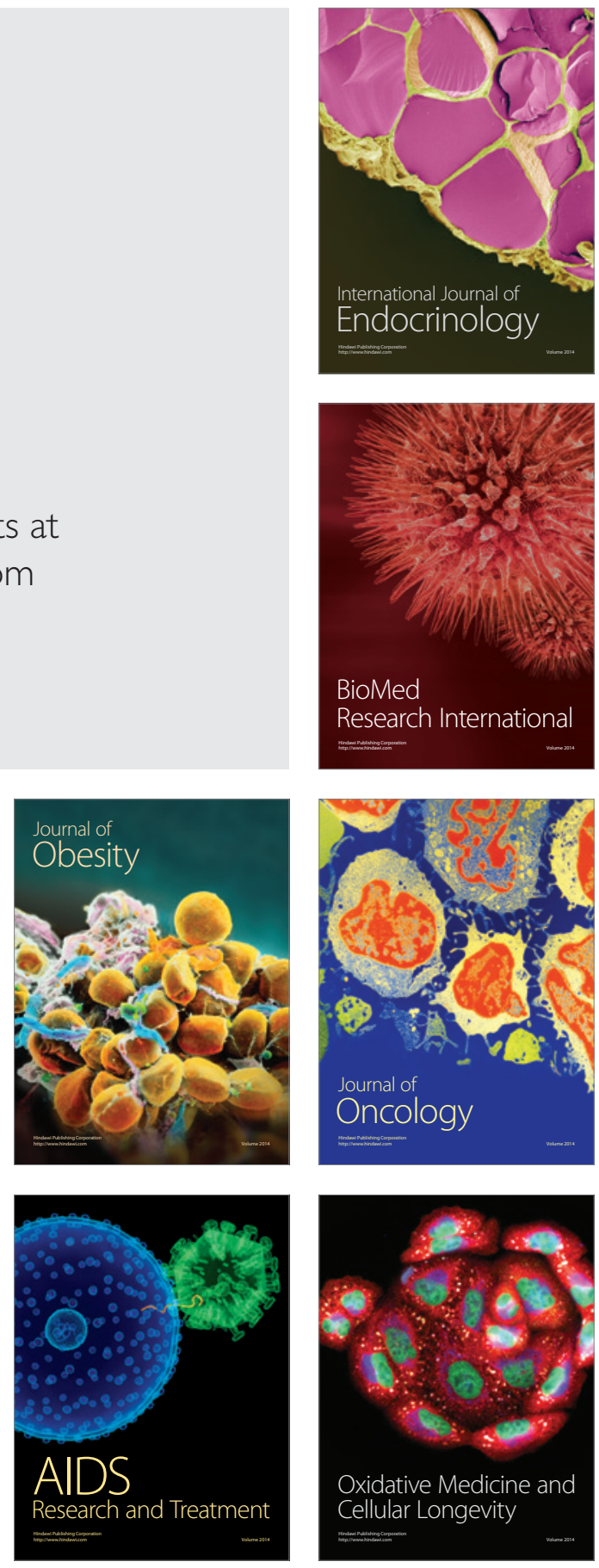